\title{
H-Singular Value of a Positive Tensor
}

\author{
Jun He \\ School of Mathematical Sciences, University of Electronic Science and Technology of China, Chengdu, China \\ Email: hejunfan1@163.com
}

Received 18 January 2015; accepted 9 February 2015; published 12 February 2015

Copyright () 2015 by author and Scientific Research Publishing Inc.

This work is licensed under the Creative Commons Attribution International License (CC BY).

http://creativecommons.org/licenses/by/4.0/

(c) (1) Open Access

\begin{abstract}
In this paper we study properties of $H$-singular values of a positive tensor $\mathcal{A} \in R^{n_{1} \times \cdots \times n_{m}}$ and present an iterative algorithm for computing the largest $\boldsymbol{H}$-singular value of the positive tensor. We prove that this method converges for any positive tensors.
\end{abstract}

\section{Keywords}

Singular Value, Positive Tensor, Convergence

\section{Introduction}

Recently, eigenvalue problems for tensors have gained special attention in the realm of numerical multilinear algebra [1]-[4], and they have a wide range of practical applications [5] [6]. The definition of eigenvalues of square tensors has been introduced in [7]-[9]. Nice properties such as the Perron-Frobenius theorem for eigenvalues of nonnegative square tensors [7] have been discussed. The authors give algorithms to compute the largest eigenvalue of a nonnegative square tensor in [6] [10]. Singular values of rectangular tensors have been introduced in [11]. In [11] [12], properties of singular values of rectangular tensors have been discussed. In particular, Chang, Qi and Zhou [11] established the Perron-Frobenius theorem to singular values of nonnegative rectangular tensors. They also proposed an iterative algorithm to find the largest singular value of a nonnegative rectangular tensor. In [13], the authors studied the convergence of the proposed algorithm.

In this paper, we focus on the tensor $\mathcal{A} \in R^{n_{1} \times \cdots \times n_{m}}$, and study properties of $H$-singular values of a positive tensor $\mathcal{A} \in R^{n_{1} \times \cdots \times n_{m}}$. For more about the definition of the $H$-singular value of a tensor $\mathcal{A} \in R^{n_{1} \times \cdots \times n_{m}}$, one can turn to the paper [14].

The paper is organized as follows. In Section 2, we recall some definitions and define $H$-singular values for a positive tensor, we extend the Perron-Frobenius theorem to $H$-singular values of positive tensors. In Section 3 , we give an algorithm to find the largest singular value of a positive tensor, some numerical experiments are given to show that our algorithm is efficient. 


\section{2. $H$-Singular Values for a Tensor}

Let $\mathcal{A} \in R^{n_{1} \times \cdots \times n_{m}}$. In this paper, we extend the definition of the classical concept of rectangular tensors, the tensors are no need square or rectangular. Consider the optimization problem

$$
\max \sum_{i_{1}}^{n_{1}} \cdots \sum_{i_{m}}^{n_{m}} a_{i_{1} \cdots i_{m}}\left(x_{1}\right)_{i_{1}} \cdots\left(x_{m}\right)_{i_{m}}, \quad x_{j} \in R^{n_{j}},
$$

under the constraints that

$$
\sum_{i=1}^{n_{1}}\left(x_{1}\right)_{i}^{m}=\cdots=\sum_{i=1}^{n_{m}}\left(x_{m}\right)_{i}^{m}=1 .
$$

We obtain the following system at a critical point:

$$
\left\{\begin{array}{c}
\mathcal{A} x_{2} \cdots x_{m}=\lambda x_{1}^{[m-1]}, \\
\vdots \\
\mathcal{A} x_{1} \cdots x_{m-1}=\lambda x_{m}^{[m-1]},
\end{array}\right.
$$

where

$$
\left\{\begin{array}{c}
\left(\mathcal{A} x_{2} \cdots x_{m}\right)_{i}=\sum_{i_{2}=1}^{n_{2}} \cdots \sum_{i_{m}}^{n_{m}} a_{i i_{2} \cdots i_{m}}\left(x_{2}\right)_{i_{2}} \cdots\left(x_{m}\right)_{i_{m}}, \\
\vdots \\
\left(\mathcal{A} x_{1} \cdots x_{m-1}\right)_{i}=\sum_{i_{1}=1}^{n_{1}} \cdots \sum_{i_{m-1}}^{n_{m-1}} a_{i_{1} \cdots i_{m-1}}\left(x_{1}\right)_{i_{1}} \cdots\left(x_{m-1}\right)_{i_{m-1}} .
\end{array}\right.
$$

If $\lambda \in C, \quad x_{j} \in C^{n_{j}} \backslash\{0\}$ are solutions of (2), then we say that $\lambda$ is an $H$-singular value of the tensor $\mathcal{A}$, $x_{1}, \cdots, x_{m}$ are eigenvectors of $\mathcal{A}$, associated with the $H$-singular value $\lambda$.

Let

$$
\begin{gathered}
P_{k}=\left\{x \in R^{k}: x_{i} \geq 0, i=1,2, \cdots, k\right\}, \\
\operatorname{int}\left(P_{k}\right)=\left\{x \in R^{k}: x_{i} \geq 0, i=1,2, \cdots, k\right\} .
\end{gathered}
$$

A vector $x \in R^{k}$ is called nonnegative if $x \in P_{k}$ and it is called strongly positive if $x \in \operatorname{int}\left(P_{k}\right)$. For any $j=1,2, \cdots, m$, let $x_{j} \in P_{n_{j}} \backslash\{0\}$ be a nonnegative vector. We give our main theorems as follows.

Lemma 1. If a tensor ${ }^{n_{j}} \mathcal{A} \in R^{n_{1} \times \cdots \times n_{m}}$ is positive, then for any $x_{j} \in P_{n_{j}} \backslash\{0\}, j=1,2, \cdots, m$,

$$
\left\{\begin{array}{c}
\mathcal{A} x_{2} \cdots x_{m}>0 \\
\vdots \\
\mathcal{A} x_{1} \cdots x_{m-1}>0
\end{array}\right.
$$

Proof. If $x_{j} \in \operatorname{int}\left(P_{n_{j}}\right), j=1,2, \cdots, m$, suppose $\mathcal{A} x_{2} \cdots x_{m}=0$, and then $a_{i_{1} \cdots i_{m}}=0$, a contradiction.

If $x_{j} \notin \operatorname{int}\left(P_{n_{j}}\right)$, and $x_{j} \neq 0, j=1,2, \cdots, m$, there exists $\left(x_{j}\right)_{i_{1}^{0}} \neq 0$, and we can get

$$
a_{i_{1} \cdots i_{m}}\left(x_{2}\right)_{i_{2}^{0}} \cdots\left(x_{m}\right)_{i_{m}^{0}}>0 \text {. }
$$

Then

$$
\mathcal{A} x_{2} \cdots x_{m}>0
$$

Similarly, we can get $\mathcal{A} x_{1} \cdots x_{m-1}>0$.

Lemma 2. Let a tensor $\mathcal{A} \in R^{n_{1} \times \cdots \times n_{m}}$ be positive, and let $\left(\lambda, x_{1}, \cdots, x_{m}\right) \in R_{+} \times \operatorname{int}\left(P_{n_{1}}\right) \times \cdots \times \operatorname{int}\left(P_{n_{m}}\right)$ be a solution of (2). If $\left(\sigma, y_{1}, \cdots, y_{m}\right) \in R_{+} \times\left(P_{n_{1}} \backslash\{0\}\right) \times \cdots \times\left(P_{n_{m}} \backslash\{0\}\right)$ satisfies

$$
\left\{\begin{array}{c}
\mathcal{A} y_{2} \cdots y_{m} \geq \sigma y_{1}^{[m-1]}, \\
\vdots \\
\mathcal{A} y_{1} \cdots y_{m-1} \geq \sigma y_{m}^{[m-1]},
\end{array}\right.
$$


Then $\sigma \leq \lambda$.

Proof. Define $t_{0}=\max \left\{s \geq 0 \mid x_{j}-s y_{j} \in P_{n_{j}}\right\}$. Since $x_{j} \in \operatorname{int} P_{n_{j}}, t_{0}>0$. We have $x_{j}-t y_{j} \geq 0$

if and only if $t \in\left[0, t_{0}\right]$. Thus

$$
\left\{\begin{array}{c}
\lambda x_{1}^{m-1}=\mathcal{A} x_{2} \cdots x_{m} \geq t_{0}^{m-1} \mathcal{A} y_{2} \cdots y_{m} \geq t_{0}^{m-1} \sigma y_{1}^{m-1} \\
\vdots \\
\lambda x_{m}^{m-1}=\mathcal{A} x_{1} \cdots x_{m-1} \geq t_{0}^{m-1} \mathcal{A} y_{1} \cdots y_{m-1} \geq t_{0}^{m-1} \sigma y_{m}^{m-1},
\end{array}\right.
$$

i.e.,

$$
\left\{\begin{array}{c}
x_{1} \geq t_{0}\left(\frac{\sigma}{\lambda}\right)^{\frac{1}{m-1}} y_{1} \\
\vdots \\
x_{m} \geq t_{0}\left(\frac{\sigma}{\lambda}\right)^{\frac{1}{m-1}} y_{m} .
\end{array}\right.
$$

This implies $\sigma \leq \lambda$.

Remark. If there exists $\left(\sigma, y_{1}, \cdots, y_{m}\right) \in R_{+} \times \operatorname{int}\left(P_{n_{1}}\right) \times \cdots \times \operatorname{int}\left(P_{n_{m}}\right)$ such that

$$
\left\{\begin{array}{c}
\mathcal{A} y_{2} \cdots y_{m} \geq \sigma y_{1}^{[m-1]}, \\
\vdots \\
\mathcal{A} y_{1} \cdots y_{m-1} \geq \sigma y_{m}^{[m-1]},
\end{array}\right.
$$

Then $\sigma$ is the eigenvalue of $\mathcal{A}$ and $y_{j}$ is the corresponding eigenvectors of $\sigma, j=1,2, \cdots, m$. This remark can be obtained by similar process in [12] [15].

Theorem 1. Assume that a tensor $\mathcal{A} \in R^{n_{1} \times \cdots \times n_{m}}$ is positive, then there exists a solution $\left(\lambda_{0}, x_{1}^{0}, \cdots, x_{m}^{0}\right)$ of system (1), satisfying $\lambda_{0}>0$ and $x_{j}^{0} \in \operatorname{int}\left(P_{n_{j}}\right)$, Moreover, if $\lambda$ is a singular value with strongly positive eigenvectors $x_{j}, j=1,2, \cdots, m$, then $\lambda=\lambda_{0}$, The strongly positive eigenvectors are unique up to a multiplicative constant,

Proof. Denote $D_{k}=\left\{z=\left(z_{1}, \cdots, z_{k}\right) \in P_{k} \mid \sum_{i=1}^{k} Z_{i}=1\right\}$. Provide by Lemma 1, the map $F$ on $D_{n_{1}} \times \cdots \times D_{n_{m}}$ into itself:

$$
F\left(\xi_{1}, \cdots, \xi_{m}\right)=\left(\frac{\left(\mathcal{A} \xi_{2} \cdots \xi_{m}\right)_{i}^{\frac{1}{m-1}}}{\sum_{i=1}^{n_{1}}\left(\mathcal{A} \xi_{2} \cdots \xi_{m}\right)_{i}^{\frac{1}{m-1}}}, \cdots, \frac{\left(\mathcal{A} \xi_{1} \cdots \xi_{m-1}\right)_{i}^{\frac{1}{m-1}}}{\sum_{i=1}^{n_{m}}\left(\mathcal{A} \xi_{1} \cdots \xi_{m-1}\right)_{i}^{\frac{1}{m-1}}}\right) .
$$

is well defined.

According to the Brouwer Fixed Point Theorem, there exists $\left(\xi_{1}^{0}, \cdots, \xi_{m}^{0}\right) \in D_{n_{1}} \times \cdots \times D_{n_{m}}$ such that

$$
\left\{\begin{array}{c}
\mathcal{A} \xi_{2}^{0} \cdots \xi_{m}^{0}=\sigma_{1}^{0} \xi_{1}^{[m-1]}, \\
\vdots \\
\mathcal{A} \xi_{1}^{0} \cdots \xi_{m-1}^{0}=\sigma_{m}^{0} \xi_{m}^{[m-1]},
\end{array}\right.
$$

where

$$
\left\{\begin{array}{c}
\sigma_{1}^{0}=\left(\sum_{i=1}^{n_{1}}\left(\mathcal{A} \xi_{2}^{0} \cdots \xi_{m}^{0}\right)_{i}^{\frac{1}{m-1}}\right)^{m-1}, \\
\vdots \\
\sigma_{m}^{0}=\left(\sum_{i=1}^{n_{m}}\left(\mathcal{A} \xi_{1}^{0} \cdots \xi_{m-1}^{0}\right)_{i}^{\frac{1}{m-1}}\right)^{m-1} .
\end{array}\right.
$$


Let

$$
x_{1}^{0}=t_{1} \xi_{1}^{0}, \cdots, x_{m}^{0}=t_{m} \xi_{m}^{0} \text {, and } \lambda_{0}=\left(\sigma_{1}^{0} \cdots \sigma_{m}^{0}\right)^{\frac{1}{m}} \text {. }
$$

Then $\left(\lambda_{0},\left(x_{1}^{0}, \cdots, x_{m}^{0}\right)\right)$ is a solution of (2).

Let us show: $\left(x_{1}^{0}, \cdots x_{m}^{0}\right) \in \operatorname{int}\left(P_{n_{1}}\right) \times \cdots \times \operatorname{int}\left(P_{n_{m}}\right)$. If not, suppose $\left(x_{j}^{0}\right)_{i}=0$, that is to say,

$$
\left(\mathcal{A} x_{1}^{0} \cdots x_{j-1}^{0} x_{j+1}^{0} \cdots x_{m}^{0}\right)_{i}=0,
$$

this contradicts the result of Lemma 1. Therefore,

$$
\left(x_{1}^{0}, \cdots, x_{m}^{0}\right) \in \operatorname{int}\left(P_{n_{1}}\right) \times \cdots \times \operatorname{int}\left(P_{n_{m}}\right) .
$$

The uniqueness of the positive singular value with strongly positive left and right eigenvectors now follows from Lemma 2 directly. The uniqueness up to a multiplicative constant of the strongly positive left and right eigenvectors is proved in the same way as in [7].

Theorem 2. Assume that $\mathcal{A} \in R^{n_{1} \times \cdots \times n_{m}}$ is a positive tensor, then

$$
\min _{x_{j} \in P_{n} \backslash\{0\}} \max _{i}\left(\frac{\left(\mathcal{A} x_{2} \cdots x_{m}\right)_{i}}{\left(x_{1}\right)_{i}^{m-1}}, \cdots, \frac{\left(\mathcal{A} x_{1} \cdots x_{m-1}\right)_{i}}{\left(x_{m}\right)_{i}^{m-1}}\right)=\lambda_{0}=\max _{x_{j} \in P_{n} \backslash\{0\}} \min _{i}\left(\frac{\left(\mathcal{A} x_{2} \cdots x_{m}\right)_{i}}{\left(x_{1}\right)_{i}^{m-1}}, \cdots, \frac{\left(\mathcal{A} x_{1} \cdots x_{m-1}\right)_{i}}{\left(x_{m}\right)_{i}^{m-1}}\right),
$$

where $\lambda_{0}$ is the unique positive singular value corresponding to strongly positive eigenvectors.

Proof. Let $x_{j} \in P_{n_{j}} \backslash\{0\}, j=1,2, \cdots, m$. We define

$$
u_{*}\left(x_{1}, \cdots, x_{m}\right)=\min _{i}\left(\frac{\left(\mathcal{A} x_{2} \cdots x_{m}\right)_{i}}{\left(x_{1}\right)_{i}^{m-1}}, \cdots, \frac{\left(\mathcal{A} x_{1} \cdots x_{m-1}\right)_{i}}{\left(x_{m}\right)_{i}^{m-1}}\right) .
$$

Since it is a positively 0-homogeneous function, it can be restricted on $D_{n_{1}} \times \cdots \times D_{n_{m}}$. Let

$$
r_{*}=u_{*}\left(x_{1}^{*}, \cdots, x_{m}^{*}\right)=\max _{x_{i} \in D_{n_{i}}} u_{*}\left(x_{1}, \cdots, x_{m}\right) .
$$

Let $\left(\lambda_{0},\left(x_{1}^{0}, \cdots, x_{m}^{0}\right)\right)$ is a solution of (2). On one hand, we have

$$
\lambda_{0}=u_{*}\left(x_{1}^{0}, \cdots, x_{m}^{0}\right) \leq u_{*}\left(x_{1}^{*}, \cdots, x_{m}^{*}\right)=r_{*} .
$$

On the other hand, by the definition of $u_{*}\left(x_{1}, \cdots, x_{m}\right)$, we get

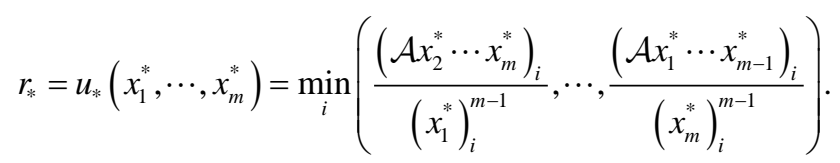

This means

$$
\left\{\begin{array}{c}
\mathcal{A} x_{2}^{*} \cdots x_{m}^{*} \geq r_{*} x_{1}^{*[m-1]} \\
\vdots \\
\mathcal{A} x_{1}^{*} \cdots x_{m-1}^{*} \geq r_{*} x_{m}^{*[m-1]} .
\end{array}\right.
$$

According to Lemma 2, we have $r_{*} \leq \lambda_{0}$, and the we get

$$
\lambda_{0}=r_{*} \text {. }
$$

Similarly, we prove the other equality.

Theorem 3. Assume that $\mathcal{A} \in R^{n_{1} \times \cdots \times n_{m}}$ is a positive tensor, and $\lambda_{0}$ is the positive singular value with strongly positive eigenvectors. Then $|\lambda| \leq \lambda_{0}$ for all singular values $\lambda$ of $\mathcal{A}$.

Proof. Let $x_{j} \in C_{n_{j}} \backslash\{0\}$ for some $\lambda \in C, j=1,2, \cdots, m$. We wish to show $|\lambda| \leq \lambda_{0}$. Let $y_{i}=\left|x_{i}\right|$. We get

$$
|\lambda|\left(y_{j}\right)_{i}^{m-1}=|\lambda|\left|x_{j}\right|_{i}^{m-1}=\left|\left(\mathcal{A} x_{1} \cdots x_{j-1} x_{j} \cdots x_{m}\right)_{i}\right| \leq\left(\mathcal{A} y_{1} \cdots y_{j-1} y_{j} \cdots y_{m}\right)_{i} .
$$


Apply Theorem 2, we can get

$$
|\lambda| \leq \max _{y_{j} \in P_{n} \backslash\{0\}} \min _{i}\left(\frac{\left(\mathcal{A} y_{2} \cdots y_{m}\right)_{i}}{\left(y_{1}\right)_{i}^{m-1}}, \cdots, \frac{\left(\mathcal{A} y_{1} \cdots y_{m-1}\right)_{i}}{\left(y_{m}\right)_{i}^{m-1}}\right)=\lambda_{0} .
$$

Theorem 4. Suppose that $\mathcal{A} \in R^{n_{1} \times \cdots \times n_{m}}$ is a positive tensor satisfying

$$
\sum_{i_{2}}^{n_{2}} \cdots \sum_{i_{m}}^{n_{m}} a_{i_{1} \cdots i_{m}}=\cdots=\sum_{i_{1}}^{n_{1}} \cdots \sum_{i_{m-1}}^{n_{m-1}} a_{i_{1} \cdots i_{m}}=C,
$$

where $C \geq 0$ is a constant. Then $\lambda_{0}(\mathcal{A})=C$.

Proof. Let $\left(\lambda_{0},\left(x_{1}^{0}, \cdots, x_{m}^{0}\right)\right)$ is a solution of (2). Without loss of generality, we suppose that $\left(x_{1}^{0}\right)_{1}=\max _{i}\left(x_{j}^{0}\right)_{i}$, $j=1, \cdots, m$. Then

$$
\begin{aligned}
\lambda_{0} & =\frac{\left(\mathcal{A} x_{2}^{0} \cdots x_{m}^{0}\right)_{1}}{\left(x_{1}^{0}\right)_{1}^{m-1}}=\frac{\left(\sum_{i_{2}=1}^{n_{2}} \cdots \sum_{i_{m}=1}^{n_{m}} a_{i_{1} \cdots i_{m}}\left(x_{2}^{0}\right)_{i_{2}} \cdots\left(x_{m}^{0}\right)_{i_{m}}\right)_{1}}{\left(x_{1}^{0}\right)_{1}^{m-1}} \\
& \leq \frac{\left(\sum_{i_{2}=1}^{n_{2}} \cdots \sum_{i_{m}=1}^{n_{m}} a_{i_{1} \cdots i_{m}}\left(x_{1}^{0}\right)_{1} \cdots\left(x_{1}^{0}\right)_{1}\right)_{1}}{\left(x_{1}^{0}\right)_{1}^{m-1}} \\
& =\sum_{i_{2}=1}^{n_{2}} \cdots \sum_{i_{m}=1}^{n_{m}} a_{i_{1} \cdots i_{m}}=C .
\end{aligned}
$$

On the other hand, it is easy to check that $C$ is an eigenvalue of $A$ with corresponding eigenvectors $\left(x_{j}\right)_{i}=1$, $j=1, \cdots, m$. So $C \leq \lambda_{0}$. Thus we have $\lambda_{0}=C$.

\section{An Iterative Algorithm}

In this section, we propose an iterative algorithm to calculate the largest $H$-singular value of a positive tensor based on Theorem $\mathbf{2}$ and Theorem 3. This algorithm is a modified version of the one given in [11] [13], and we will show the convergence of the proposed algorithm for any positive tensor. In this section, we always suppose that $\mathcal{A}$ is a positive tensor.

For a positive tensor $\mathcal{A} \in R^{n_{1} \times \cdots \times n_{m}}, \rho>0, \quad x_{j} \in P_{n_{j}}$, let

$$
\left\{\begin{aligned}
\eta_{1}\left(x_{1}, \cdots, x_{m}\right) & =\mathcal{A} x_{2} \cdots x_{m}+\rho x_{1}^{[m-1]}, \\
\vdots & \\
\eta_{m}\left(x_{1}, \cdots, x_{m}\right) & =\mathcal{A} x_{1} \cdots x_{m-1}+\rho x_{m}^{[m-1]} .
\end{aligned}\right.
$$

\section{Algorithm 3.1}

Step 0 Choose $x_{1}^{(1)}>0, \cdots, x_{m}^{(1)}>0$. Set $k=1$;

Step 1 Compute

$$
\left\{\begin{array}{c}
\eta_{1}^{(k)}=\eta_{1}\left(x_{1}^{k}, \cdots, x_{m}^{k}\right), \\
\vdots \\
\eta_{m}^{(k)}=\eta_{m}\left(x_{1}^{k}, \cdots, x_{m}^{k}\right) .
\end{array}\right.
$$

Let

$$
\begin{aligned}
& \underline{u}_{k}=\min _{\left(x_{j}^{(k)}\right)_{i}>0}\left\{\frac{\left(\eta_{1}^{(k)}\right)_{i}}{\left(x_{1}^{(k)}\right)_{i}^{m-1}}, \cdots, \frac{\left(\eta_{m}^{(k)}\right)_{i}}{\left(x_{m}^{(k)}\right)_{i}^{m-1}}\right\}, \\
& \bar{u}_{k}=\max _{\left(x_{j}^{(k)}\right)_{i}>0}\left\{\frac{\left(\eta_{1}^{(k)}\right)_{i}}{\left(x_{1}^{(k)}\right)_{i}^{m-1}}, \cdots, \frac{\left(\eta_{m}^{(k)}\right)_{i}}{\left(x_{m}^{(k)}\right)_{i}^{m-1}}\right\} ;
\end{aligned}
$$

Step 2 If $\underline{u}_{k}=\bar{u}_{k}$, then stop. Otherwise, compute 


$$
\left\{\begin{array}{c}
x_{1}^{(k+1)}=\frac{\left(\eta_{1}^{(k)}\right)^{\left[\frac{1}{m-1}\right]}}{\|\left(\eta_{1}^{(k)}, \cdots, \eta_{m}^{(k)}\right)^{\left[\frac{1}{m-1}\right] \|}} \\
\vdots \\
x_{m}^{(k+1)}=\frac{\left(\eta_{m}^{(k)}\right)^{\left[\frac{1}{m-1}\right]}}{\|\left(\eta_{1}^{(k)}, \cdots, \eta_{m}^{(k)}\right)^{\left[\frac{1}{m-1}\right] \|}},
\end{array}\right.
$$

and replace $k$ by $k+1$ and go to Step 1 .

In the following, we will give a convergence result for Algorithm 3.1.

Theorem 5. Assume that $\left(u_{0},\left(x_{1}^{0}, \cdots, x_{m}^{0}\right)\right)$ is a solution of (2). Then,

$$
0 \leq \underline{u}_{1} \leq \underline{u}_{2} \leq \cdots \leq u_{0} \leq \cdots \leq \bar{u}_{2} \leq \bar{u}_{1} .
$$

Proof. By (8), $0<\underline{u}_{1}$. From Theorem 2, for $k=1,2, \cdots$,

$$
\underline{u}_{k} \leq u_{0} \leq \bar{u}_{k} \text {. }
$$

We now prove for any $k \geq 1$,

$$
\underline{u}_{k} \leq \underline{u}_{k+1}, \quad \bar{u}_{k} \leq \bar{u}_{k+1} .
$$

For each $k=1,2, \cdots$, by the definition of $\underline{u}_{k}$ and Lemma 1, we have

$$
\eta_{1}^{(k)} \geq \underline{u}_{k}\left(x_{1}^{(k)}\right)^{[m-1]}>0, \cdots, \eta_{m}^{(k)} \geq \underline{u}_{k}\left(x_{m}^{(k)}\right)^{[m-1]}>0 .
$$

Then,

$$
\left(\eta_{1}^{(k)}\right)^{\left[\frac{1}{m-1}\right]} \geq\left(\underline{u}_{k}\right)^{\frac{1}{m-1}} x_{1}^{(k)}>0, \cdots,\left(\eta_{m}^{(k)}\right)^{\left[\frac{1}{m-1}\right]} \geq\left(\underline{u}_{k}\right)^{\frac{1}{m-1}} x_{m}^{(k)}>0 .
$$

So,

Hence, we get

$$
\begin{aligned}
& x_{1}^{(k+1)}=\frac{\left(\eta_{1}^{(k)}\right)^{\left[\frac{1}{m-1}\right]}}{\left\|\left(\eta_{1}^{(k)}, \cdots, \eta_{m}^{(k)}\right)^{\left[\frac{1}{m-1}\right]}\right\|} \geq \frac{\left(\underline{u}_{k}\right)^{\frac{1}{m-1}} x_{1}^{(k)}}{\left\|\left(\eta_{1}^{(k)}, \cdots, \eta_{m}^{(k)}\right)^{\left[\frac{1}{m-1}\right]}\right\|}>0, \\
& x_{m}^{(k+1)}=\frac{\left(\eta_{m}^{(k)}\right)^{\left[\frac{1}{m-1}\right]}}{\left\|\left(\eta_{1}^{(k)}, \cdots, \eta_{m}^{(k)}\right)^{\left[\frac{1}{m-1}\right]}\right\|} \geq \frac{\left(\underline{u}_{k}\right)^{\frac{1}{m-1}} x_{m}^{(k)}}{\left\|\left(\eta_{1}^{(k)}, \cdots, \eta_{m}^{(k)}\right)^{\left[\frac{1}{m-1}\right]}\right\|}>0 .
\end{aligned}
$$

$$
\begin{gathered}
\eta_{1}^{(k+1)} \geq \frac{\underline{u}_{k} \eta_{1}^{(k)}}{\left\|\left(\eta_{1}^{(k)}, \cdots, \eta_{m}^{(k)}\right)^{\frac{1}{m-1}}\right\|^{m-1}}=\underline{u}_{k}\left(x_{1}^{(k+1)}\right)^{m-1}, \\
\vdots \\
\eta_{m}^{(k+1)} \geq \frac{\underline{u}_{k} \eta_{1}^{(k)}}{\left\|\left(\eta_{1}^{(k)}, \cdots, \eta_{m}^{(k)}\right)^{\frac{1}{m-1}}\right\|^{m-1}}=\underline{u}_{k}\left(x_{m}^{(k+1)}\right)^{m-1},
\end{gathered}
$$


which means for $j=1,2, \cdots, m$

$$
\underline{u}_{k} \leq \frac{\left(\eta_{j}^{(k+1)}\right)_{i}}{\left(x_{j}^{(k+1)}\right)_{i}^{m-1}} .
$$

Therefore, we get

$$
\underline{u}_{k} \leq \underline{u}_{k+1} .
$$

Similarly, we can prove that

$$
\bar{u}_{k} \leq \bar{u}_{k+1} .
$$

From Theorem 5, $\left\{\underline{u}_{k}\right\}$ is a monotonic increasing sequence and it has an upper bound, so the limit exists. Since $\left\{\bar{u}_{k}\right\}$ is monotonic decreasing sequence and it has a lower bound, the limit exists as well. We suppose

$$
\underline{u}=\lim _{k \rightarrow \infty} \underline{u}_{k}, \quad \bar{u}=\lim _{k \rightarrow \infty} \bar{u}_{k} .
$$

By Theorem 5, we have

$$
0<\underline{u} \leq u_{0} \leq \bar{u} .
$$

The argument used in the following proof is parallel to that in [13]. We proceed the proof for completeness.

Theorem 6. Let $\left\{x_{j}^{(k)}\right\},\left\{\eta_{j}^{(k)}\right\} \quad(j=1,2, \cdots, m)$ be the sequences produced by Algorithm 3.1. Then

a) $\left\{x_{j}^{(k)}\right\}$ have convergent subsequences which converge to $x_{j}^{*}$, respectively. Moreover, $x_{j}^{*} \in P_{n_{j}} \backslash\{0\}$, $j=1,2, \cdots, m$.

b) $\eta_{1}\left(x_{2}^{*}, \cdots, x_{m}^{*}\right) \geq \underline{u}\left(x_{1}^{*}\right)^{m-1}, \cdots, \eta_{m}\left(x_{1}^{*}, \cdots, x_{m-1}^{*}\right) \geq \underline{u}\left(x_{m}^{*}\right)^{m-1}$.

c) $\underline{u}=u_{0}=\bar{u}$.

Proof. As $\left\|\left(x_{1}^{(k)}, \cdots, x_{m}^{(k)}\right)\right\|=1$ for all $k \geq 2$. Hence, there exists a convergent subsequence by the compactness of the unit ball in $R^{n}$ and $x_{j}^{*}$ must not be a zero vector.

By the continuity of $\eta_{j},(8)$ and (9), we get the result (b).

If $\underline{u} \neq \bar{u}$, we get that someone of the follow inequations exists:

$\eta_{1}\left(x_{2}^{*}, \cdots, x_{m}^{*}\right) \neq \underline{u}\left(x_{1}^{*}\right)^{m-1}, \cdots \eta_{m}\left(x_{1}^{*}, \cdots, x_{m-1}^{*}\right) \neq \underline{u}\left(x_{m}^{*}\right)^{m-1}$. By Theorem 2.5 in [13], there exists a positive integer $s$ such that

$$
\underline{u} \eta_{j}^{s}\left(x_{1}^{*}, \cdots, x_{m}^{*}\right)<\eta_{j}^{s}\left(\left(\eta_{1}^{*}\right)^{\frac{1}{m-1}}, \cdots,\left(\eta_{m}^{*}\right)^{\frac{1}{m-1}}\right) .
$$

By (a) and the continuity of $\eta_{j}$, for any sufficiently large $k_{j}$, we obtain

$$
\underline{u} \eta_{j}^{s}\left(x_{1}^{k_{j}}, \cdots, x_{m}^{k_{j}}\right)<\eta_{j}^{s}\left(\left(\eta_{1}^{k_{j}}\right)^{\frac{1}{m-1}}, \cdots,\left(\eta_{m}^{k_{j}}\right)^{\frac{1}{m-1}}\right) .
$$

Then we obtain $\underline{u}_{k_{j}+s}>\underline{u}$, which contradicts with Theorem 5. So (c) holds.

By Theorem 6, we can get the largest $H$-singular value of $\mathcal{A}$ is

$$
\lambda_{0}=u_{0}-\rho .
$$

In the following, in order to show the viability of Algorithm 3.1, we used Matlab 7.1 to test it with some randomly generated rectangular tensors. For these randomly generated tensors, the value of each entry is between 0 and 10. we set $x_{1}^{(1)}=[1,1, \cdots, 1]^{\mathrm{T}} \in R^{n_{1}}, \cdots, x_{m}^{(1)}=[1,1, \cdots, 1]^{\mathrm{T}} \in R^{n_{m}}$. We terminated our iteration when $\bar{\lambda}_{k}-\underline{\lambda}_{k} \leq 10^{-6}$.

Our numerical results are shown in Table 1. In this table, Ite denotes the number of iterations, $\bar{\lambda}-\underline{\lambda}$ and $\lambda$ denote the values of $\bar{\lambda}_{k}-\underline{\lambda}_{k}$ and $0.5\left(\bar{\lambda}_{k}+\underline{\lambda}_{k}\right)$ at the final iteration, respectively. $\operatorname{Norm} X_{j}(j=1,2, \cdots, m)$ denote the values of at the final iteration, respectively. The results in Table 1 show that the proposed algorithm is promising. The algorithm is able to produce the largest singular values for all these randomly generated posi- 
Table 1. Numerical results of Algorithm 3.1 for randomly generated tensors.

\begin{tabular}{ccccccc}
\hline$\left(n_{1}, n_{2}, n_{3}\right)$ & Ite & $\bar{\lambda}-\underline{\lambda}$ & $\lambda$ & Norm $X_{1}$ & Norm $X_{2}$ & Norm $X_{3}$ \\
\hline $2 \times 3 \times 3$ & 26 & $8.95 \mathrm{e}-007$ & 36.78 & $2.42 \mathrm{e}-008$ & $1.96 \mathrm{e}-008$ & $1.87 \mathrm{e}-008$ \\
$2 \times 3 \times 4$ & 27 & $7.68 \mathrm{e}-007$ & 41.08 & $1.18 \mathrm{e}-008$ & $8.30 \mathrm{e}-009$ & $8.86 \mathrm{e}-009$ \\
$2 \times 3 \times 5$ & 28 & $6.10 \mathrm{e}-007$ & 46.39 & $2.82 \mathrm{e}-009$ & $2.44 \mathrm{e}-009$ & $1.87 \mathrm{e}-009$ \\
$2 \times 3 \times 10$ & 29 & $9.24 \mathrm{e}-007$ & 77.87 & $2.16 \mathrm{e}-009$ & $1.71 \mathrm{e}-009$ & $8.89 \mathrm{e}-010$ \\
$3 \times 6 \times 10$ & 30 & $7.27 \mathrm{e}-007$ & 165.51 & $6.59 \mathrm{e}-009$ & $4.04 \mathrm{e}-009$ & $3.57 \mathrm{e}-009$ \\
\hline
\end{tabular}

tive tensors.

\section{Conclusion}

In this paper, we give some eigenvalues properties about the $H$-singular value of a positive tensor $\mathcal{A} \in R^{n_{1} \times \cdots \times n_{m}}$ introduced in [6]. We find that the Perron-Frobenius like theorem for nonnegative square tensors can not be extended to the nonnegative tensor $\mathcal{A} \in R^{n_{1} \times \cdots \times n_{m}}$, so here we limit the tensor $\mathcal{A} \in R^{n_{1} \times \cdots \times n_{m}}$ to the positive case. An algorithm is given to compute the largest $H$-singular value of the positive tensor.

\section{Acknowledgements}

I thank the editor and the referee for their comments. The author is funded by the Fundamental Research Funds for Central Universities.

\section{References}

[1] Chang, K.-C., Pearson, K. and Zhang, T. (2011) Primitivity, the Convergence of the NZQ Method, and the Largest Eigenvalue for Nonnegative Tensors. SIAM Journal on Matrix Analysis and Applications, 32, 806-819. http://dx.doi.org/10.1137/100807120

[2] Qi, L.Q. (2007) Eigenvalues and Invariants of Tensor. Journal of Mathematical Analysis and Applications, 325, 1363-1377.http://dx.doi.org/10.1016/j.jmaa.2006.02.071

[3] Pearson, K.J. (2010) Primitive Tensors and Convergence of an Iterative Process for the Eigenvalue of a Primitive Tensor. http://arxiv.org/abs/1004.2423

[4] Wang, Y.J., Qi, L.Q. and Zhang, X.Z. (2009) A Practical Method for Computing the Largest M-Eigenvalue of a FourthOrder Partially Symmetric Tensor. Numerical Linear Algebra with Applications, 16, 589-601. http://dx.doi.org/10.1002/nla.633

[5] Qi, L.Q., Wang, Y.J. and Wu, E.X. (2008) D-Eigenvalues of Diffusion Kurtosis Tensor. Journal of Computational and Applied Mathematics, 221, 150-157. http://dx.doi.org/10.1016/j.cam.2007.10.012

[6] Ng, M., Qi, L.Q. and Zhou, G.L. (2009) Finding the Largest Eigenvalue of a Non-Negative Tensor. SIAM Journal on Matrix Analysis and Applications, 31, 1090-1099. http://dx.doi.org/10.1137/09074838X

[7] Chang, K.-C., Pearson, K. and Zhang, T. (2008) Perron-Frobenius Theorem for Nonnegative Tensors. Communications in Mathematical Sciences, 6, 507-520. http://dx.doi.org/10.4310/CMS.2008.v6.n2.a12

[8] Chang, K.-C., Pearson, K. and Zhang, T. (2009) On Eigenvalue Problems of Real Symmetric Tensors. Journal of Mathematical Analysis and Applications, 350, 416-422. http://dx.doi.org/10.1016/j.jmaa.2008.09.067

[9] Qi, L.Q. (2005) Eigenvalues of a Real Supersymmetric Tensor. Journal of Symbolic Computation, 40, 1302-1324. http://dx.doi.org/10.1016/j.jsc.2005.05.007

[10] Liu, Y.J., Zhou, G.L. and Ibrahim, N.F. (2010) An Always Convergent Algorithm for the Largest Eigenvalue of an Irreducible Nonnegative Tensor. Journal of Computational and Applied Mathematics, 235, 286-292. http://dx.doi.org/10.1016/j.cam.2010.06.002

[11] Chang, K.C., Qi, L.Q. and Zhou, G.L. (2010) Singular Values of a Real Rectangular Tensor. Journal of Mathematical Analysis and Applications, 370, 284-294. http://dx.doi.org/10.1016/j.jmaa.2010.04.037

[12] Yang, Y. and Yang, Q. (2011) Singular Values of Nonnegative Rectangular Tensors. Frontiers of Mathematics in China, 6, 363-378.

[13] Zhou, G.K., Caccetta, L. and Qi, L.Q. (2013) Convergence of an Algorithm for the Largest Singular Value of a Nonnegative Rectangular Tensor. Linear Algebra and Its Applications, 438, 959-968. http://dx.doi.org/10.1016/j.laa.2011.06.038 
[14] Lim, L.H. (2005) Singular Values and Eigenvalues of Tensors: A Variational Approach. Proceedings of the IEEE International Workshop on Computational Advances in Multi-Sensor Adaptive Processing (CAMSAP’05), 1, 129-132.

[15] Yang, Y.N. and Yang, Q.Z. (2010) Further Results for Perron-Frobenius Theorem for Nonnegative Tensors. SIAM Journal on Matrix Analysis and Applications, 31, 2517-2530. http://dx.doi.org/10.1137/090778766 
Scientific Research Publishing (SCIRP) is one of the largest Open Access journal publishers. It is currently publishing more than 200 open access, online, peer-reviewed journals covering a wide range of academic disciplines. SCIRP serves the worldwide academic communities and contributes to the progress and application of science with its publication.

Other selected journals from SCIRP are listed as below. Submit your manuscript to us via either submit@scirp.org or Online Submission Portal.
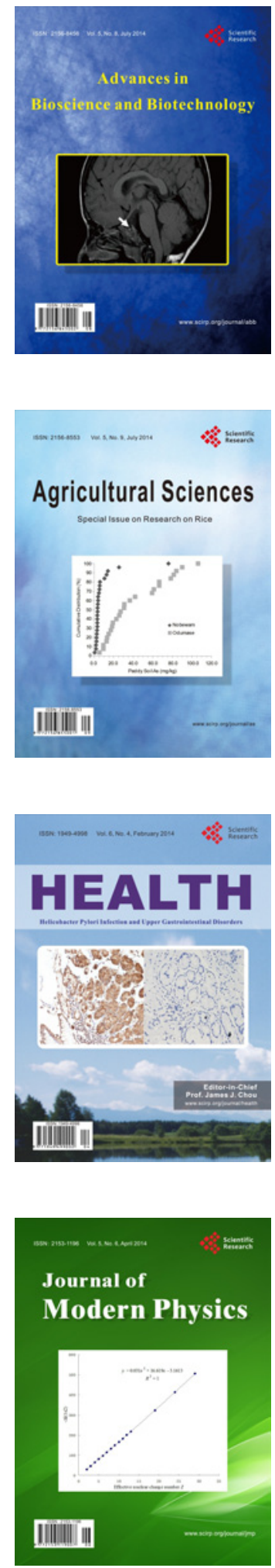
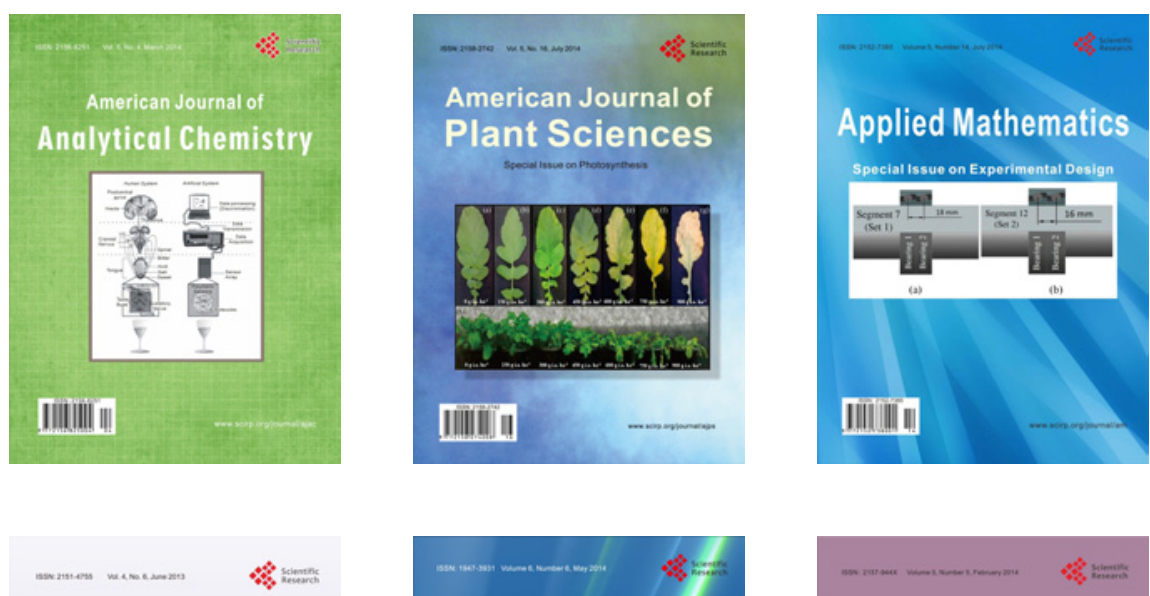

Creative Education
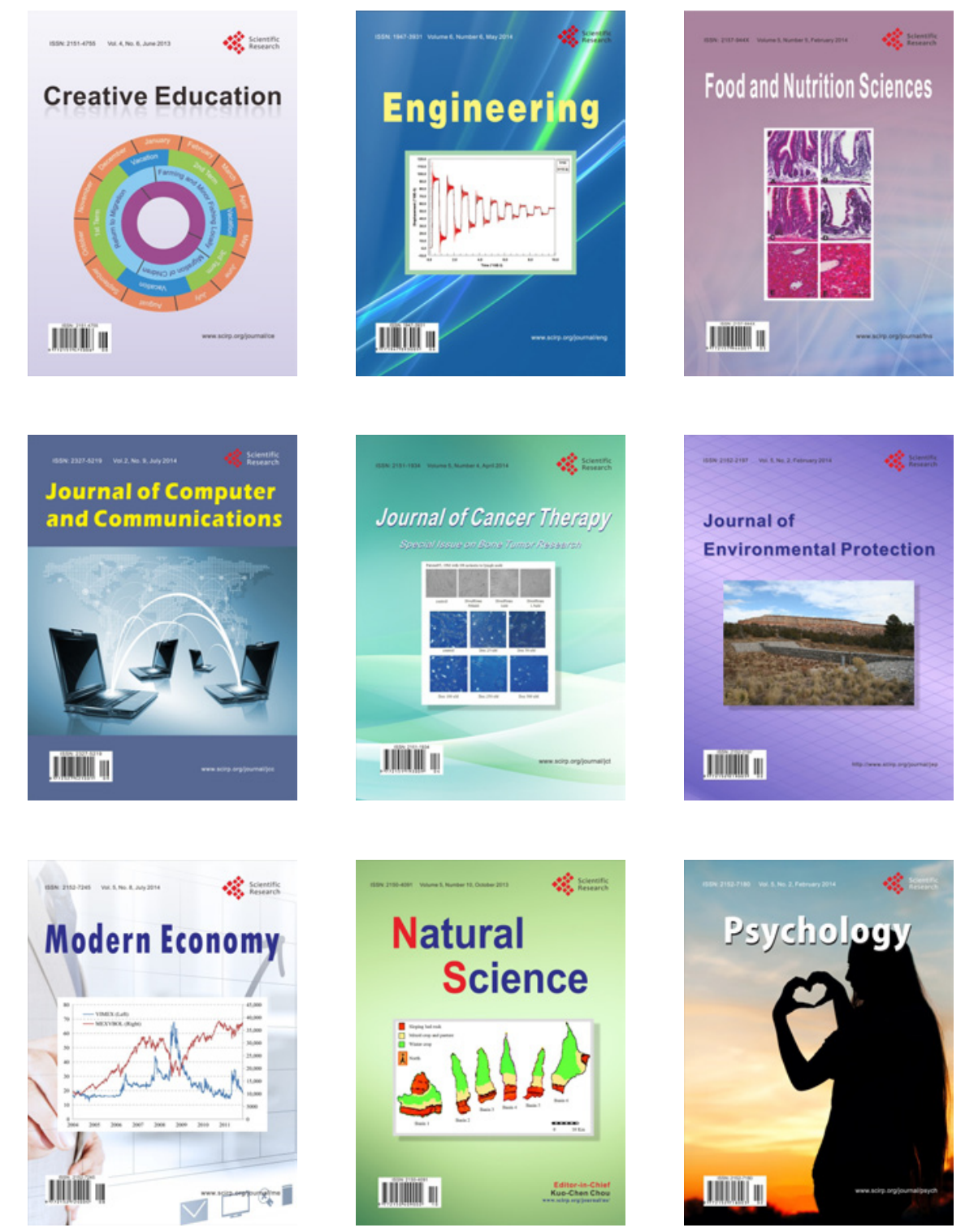